\title{
A design of HTM spatial pooler for face recognition using memristor-CMOS hybrid circuits
}

Timur Ibrayev, Alex Pappachen James, Cory Merkel, Dhireesha Kudithipudi

- School of Engineering

\begin{abstract}
Hierarchical Temporal Memory (HTM) is a machine learning algorithm that is inspired from the working principles of the neocortex, capable of learning, inference, and prediction for bit-encoded inputs. Spatial pooler is an integral part of HTM that is capable of learning and classifying visual data such as objects in images. In this paper, we propose a memristor-CMOS circuit design of spatial pooler and exploit memristors capabilities for emulating the synapses, where the strength of the weights is represented by the state of the memristor. The proposed design is validated on a challenging application of single image per person face recognition problem using AR database resulting in a recognition accuracy of $80 \%$.
\end{abstract}

Original language

Title of host publication

Publisher

Pages

Number of pages

Volume

ISBN (Electronic)

State

Event

Conference

Country

City

Period
English

ISCAS 2016 - IEEE International Symposium on Circuits and Systems

Institute of Electrical and Electronics Engineers Inc.

$1254-1257$

4

2016-July

9781479953400

Published - Jul 292016

2016 IEEE International Symposium on Circuits and Systems, ISCAS 2016 Montreal, Canada

2016 IEEE International Symposium on Circuits and Systems, ISCAS 2016

Canada

Montreal

$5 / 22 / 16 \rightarrow 5 / 25 / 16$ 
Ibrayev, T., James, A. P., Merkel, C., \& Kudithipudi, D. (2016). A design of HTM spatial pooler for face recognition using memristor-CMOS hybrid circuits. In ISCAS 2016 - IEEE International Symposium on Circuits and Systems. (Vol. 2016-July, pp. 1254-1257). [7527475] Institute of Electrical and Electronics Engineers Inc.. DOI: 10.1109/ISCAS.2016.7527475 